\title{
CSF Neurofilament Light Chain Elevation Predicts ALS Severity and Progression
}

\author{
Qionghua Sun ${ }^{1,2}$, Xue Zhao ${ }^{1,2}$, Siyuan $\mathrm{Li}^{1}$, Fei Yang ${ }^{1}$, Hongfen Wang ${ }^{1}$, Fang Cui ${ }^{3}$ and \\ Xusheng Huang ${ }^{1,2 *}$
}

${ }^{1}$ Neurological Department of the First Medical Center, Chinese PLA General Hospital, Beijing, China, ${ }^{2}$ College of Medicine, Nankai University, Tianjin, China, ${ }^{3}$ Neurological Department of Hainan Hospital, Chinese PLA General Hospital, Sanya, China

OPEN ACCESS

Edited by:

Angelo Schenone,

University of Genoa, Italy

Reviewed by:

Isabella Laura Simone,

University of Bari Aldo Moro, Italy

Sergio Ferrari,

University of Verona, Italy

*Correspondence:

Xusheng Huang

lewish301huang@163.com

Specialty section

This article was submitted to Neuromuscular Diseases,

a section of the journal

Frontiers in Neurology

Received: 19 May 2020

Accepted: 16 July 2020

Published: 28 August 2020

Citation:

Sun $Q$, Zhao X, Li S, Yang F, Wang $H$, Cui F and Huang $X$ (2020) CSF Neurofilament Light Chain Elevation Predicts ALS Severity and

Progression. Front. Neurol. 11:919. doi: 10.3389/fneur.2020.00919
Objectives: This study compared neurofilament light chain (NFL) levels in the cerebrospinal fluid (CSF) of patients with sporadic amyotrophic lateral sclerosis (sALS) with levels in patients with other neurological diseases and healthy controls and assessed correlations between NFL levels and clinical indicators of SALS.

Methods: We used enzyme-linked immunosorbent assays to determine the NFL levels in the CSF of 45 patients with SALS, 21 patients with other central nervous system diseases (OCNSDs), 18 with immune-mediated peripheral neuropathy (IMPN), 14 with non-immune-mediated peripheral neuropathy (NIMPN), and 19 healthy controls (HCs).

Results: The median NFL levels in the CSF of the SALS, OCNSD, IMPN, NIMPN, and HC groups were 6510, 5372, 4320, 1477, and 756 pg/mL, respectively. The CSF NFL levels did not differ significantly among the SALS, IMPN, and OCNSD groups, but were significantly higher than those of the NIMPN and HC groups. The NFL CSF levels were significantly higher in the NIMPN group than the HCs. There was a negative correlation between the NFL level and ALS function score (ALSFRS-R), and a positive correlation with the disease progression rate in patients with SALS.

Conclusion: CSF NFL may not be sufficient to distinguish ALS from other central nervous system diseases or peripheral neuropathy, but it predicts ALS severity and progression.

Keywords: amyotrophic lateral sclerosis, neurofilament light chain, NFL, CSF, axonal damage

\section{INTRODUCTION}

Amyotrophic lateral sclerosis (ALS) is a fatal neurodegenerative disease that selectively involves the upper and lower motor neurons. The pathogenesis of ALS remains unclear, but axonal injury may be involved (1). The diagnosis of ALS is based mainly on clinical features, electromyography, and the exclusion of other diseases. Many recent studies have focused on the role of neurofilaments (NFs) as biomarkers in ALS (2). NFs are cytoskeletal proteins and their levels increase in biological fluids proportionally to the degree of axon damage. NFs consist of light (NFL), medium (NFM), and heavy (NFH) chain subunits. There is growing evidence that the levels of NFs, especially phosphorylated neurofilament heavy chains (p-NFH) and neurofilament light chains (NFL), reflect axonal injury and are potentially of value in ALS and other neurological disorders with axon damage. Cerebrospinal fluid (CSF) and blood levels of NFs are increased in ALS (3-13) and 
other neurological disorders, including Alzheimer's disease $(14,15)$, multiple sclerosis $(16,17)$, multiple system atrophy predominated by parkinsonism (18), Charcot-Marie-Tooth disease (19), and cerebrovascular disease (20). NFs are non-specific markers of axon damage. To determine whether NFL can distinguish ALS from other nervous system diseases, we determined the NFL levels in CSF from patients with sALS and compared the levels in other nervous system diseases. Furthermore, some studies have observed that NFL levels were correlated with the clinical upper motor neuron burden $(7,21,22)$, and increased levels were associated with a poor prognosis of ALS (23-27). Therefore, we also assessed the relationship between NFL levels and clinical indicators of sALS.

\section{MATERIALS AND METHODS}

\section{Patients}

The study enrolled 45 patients diagnosed with sALS admitted to the Department of Neurology at the Chinese PLA General Hospital from December 2017 to December 2018. The diagnosis was based on the Revised E1 Escorial diagnostic criteria (28). Three disease control groups were also enrolled: 20 patients with other central nervous system diseases (OCNSDs) [seven cases of multiple system atrophy (MSA), four of subacute combined degeneration (SCD), two of multiple sclerosis (MS), and one case each of corticobasal degeneration, spasmodic torticollis, progressive supranuclear palsy, spinocerebellar ataxia, Alzheimer's disease, adrenoleukodystrophy, and acute myelitis]; 18 patients with immune-mediated peripheral neuropathy (IMPN) [five with chronic inflammatory demyelinating polyradiculoneuropathy (CIDP), three with Guillain-Barré syndrome (GBS), one with chronic idiopathic axonal polyneuropathy, and nine with other immune-mediated peripheral neuropathies]; and 14 patients with non-immunemediated peripheral neuropathy (NIMPN), such as metabolic and hereditary peripheral neuropathy. Another 19 patients with primary migraine and somatization disorder were enrolled as healthy controls (HCs). Participants were excluded from the study if they had brain trauma, cerebral infarction, or other conditions that alter CSF NFL levels (29).

\section{Clinical Parameters of ALS and Sample Collection}

The clinical data of the 45 patients with sALS were recorded, including age, gender, site of symptom onset, disease duration (time from symptom onset to sample collection), diagnostic category (clinically definite, clinically probable, clinically probable-laboratory-supported, or clinically possible ALS), and the time of symptom spread from spinal or bulbar localization to generalization (TTG), which is an early clinical parameter of disease progression (30), as well as clinical phenotypes, including upper (UMND-ALS) and lower (LMND-ALS) motor neuron dominant ALS or classical ALS, according to the extent of UMN and LMN involvement and areas affected the time of sampling (31). The onset site was classified as bulbar or limb onset. We also determined the score on the ALS functional rating scale revised (ALSFRS-r) (32). The disease progression rate (DPR) was calculated as [(48 - ALSFRS-r score at evaluation)/(disease duration from symptom onset to evaluation in months)] (33). The forced vital capacity (FVC) of the ALS patients was also tested.

CSF samples were collected from all patients and controls at the time of diagnosis in our department after lumbar puncture for CSF analysis. All samples were centrifuged at 3,000 rpm at $4^{\circ} \mathrm{C}$ for $10 \mathrm{~min}$ within $2 \mathrm{~h}$ of collection. The supernatants were stored at $-80^{\circ} \mathrm{C}$ until tested. This study was approved by the ethics committee of the Chinese PLA General Hospital. Informed consent was obtained from all participants.

\section{NFL ELISA}

The NFL CSF concentrations were measured using commercial enzyme-linked immunosorbent assays (ELISAs; IBL International, Hamburg, Germany), performed in accordance with the manufacturer's instructions. The range 100-10,000 $\mathrm{ng} / \mathrm{L}$ was explored. Samples were run in duplicate, together with freshly prepared standards, and positive and negative controls on each plate.

\section{Statistical Analysis}

For continuous variables, the Kolmogorov-Smirnov test was used to determine whether the data were normally distributed and Levene's test to determine the homogeneity of the variance. Normally distributed data are expressed as the mean \pm standard deviation and non-normally distributed data as the median and interquartile range (IQR). The CSF NFL levels were non-normally distributed. Differences in the CSF NFL levels between the HCs and sALS or OCNSD groups were determined by analysis of covariance adjusted for age. Wilcoxon's nonparametric test was used to compare the NFL levels among the other groups. Spearman correlation analysis was used to analyze the associations between the CSF NFL level and clinical parameters in the sALS patients. The analyses were performed using SPSS (ver. 20.0; SPSS, Chicago, IL, USA). The level of statistical significance was set at $p<0.05$.

\section{RESULTS}

\section{Demographic Characteristics of the Subjects}

Table 1 shows the age and gender of all subjects. The NFL concentration in all subjects was significantly correlated with age $(r=0.339, p<0.0001)$, but not with gender $(r=0.060$, $p=0.518)$. Among the five groups, significant differences were found in age between the HCs and sALS $(p=0.016)$ and OCNSD $(p=0.010)$ groups. To avoid age-related bias when comparing NFL levels, differences in CSF NFL levels between the HCs and sALS and OCNSD groups were analyzed using analysis of covariance after adjusting for age (Table $\mathbf{1}$ ).

\section{Clinical Parameters of the ALS Group}

The study enrolled 45 patients with sALS (25 males, 20 females). Of these, 28 were diagnosed with clinically definite ALS, 15 with clinically probable ALS, and two with clinically possible ALS. 
TABLE 1 | Demographic characteristics of the study groups.

\begin{tabular}{lccc}
\hline Group & $\boldsymbol{n}$ & Male/Female (n) & Mean age, years (SD) \\
\hline SALS & 45 & $25 / 20$ & $54.29(8.81)$ \\
OCNSD & 20 & $16 / 4$ & $56.75(13.76)$ \\
IMPN & 18 & $12 / 6$ & $52.06(15.43)$ \\
NIMPN & 14 & $10 / 4$ & $45.21(14.13)$ \\
HC & 19 & $13 / 6$ & $40.11(16.28)$ \\
\hline
\end{tabular}

ALS, amyotrophic lateral sclerosis; OCNSD, other central nervous system diseases; IMPN, immune-mediated peripheral neuropathy; NIMPN, non-immune-mediated peripheral neuropathy; $\mathrm{HC}$, healthy control; $S D$, standard deviation.

TABLE 2 | NFL levels in CSF of patients from different groups.

\begin{tabular}{lcccccc}
\hline $\boldsymbol{p}$-value & NFL(pg/ml) & sALS & OCNSD & IMPN & NIMPN & HC \\
\hline SALS & $6510(4557-8598)$ & $/$ & 0.091 & 0.132 & $<0.0001$ & $<0.0001$ \\
OCNSD & $5307(3413-7181)$ & 0.091 & $/$ & 0.520 & $<0.0001$ & $<0.0001$ \\
IMPN & $4320(1948-9183)$ & 0.132 & 0.520 & $/$ & 0.001 & $<0.0001$ \\
NIMPN & $1477(913-1875)$ & $<0.0001$ & $<0.0001$ & 0.001 & $/$ & 0.001 \\
HC & $756(551-960)$ & $<0.0001$ & $<0.0001$ & $<0.0001$ & 0.001 & $/$
\end{tabular}

CSF, cerebrospinal fluid; NFL, neurofilament light chain; ALS, amyotrophic lateral sclerosis; OCNSD, other central nervous system diseases; IMPN, immune-mediated peripheral neuropathy; NIMPN, non-immune-mediated peripheral neuropathy; HC, healthy control. NFL levels are expressed as median values (interquartile range). The $p$-value in bold was $<0.05$, indicating that the NFL levels of the corresponding two groups were statistically different.

Eight cases were classified as bulbar onset and 37 as limb onset ALS. The mean age at sampling was $54.29 \pm 8.81$ years. The median disease duration was 12 (range 3-84) months. There were 37 patients with classical ALS and four each with UMND-ALS and LMND-ALS. The mean ALSFRS-r score was $37.29 \pm 8.54$ and the mean DPR was $0.94 \pm 0.85$. The mean FVC\%, which was obtained from 39 ALS patients, was $87.23 \pm 21.17 \%$. Twentytwo cases progressed to generalized ALS and the mean TTG was $12.18 \pm 11.30$ months.

\section{Comparison of NFL Levels Between ALS Patients and Controls}

The median CSF NFL levels in the sALS, OCNSD, IMPN, NIMPN, and HC groups were 6510, 5307, 4320, 1477, and $756 \mathrm{pg} / \mathrm{mL}$, respectively (Table 1). No significant differences were observed in NFL levels among the sALS, OCNSD, and IMPN groups. The CSF NFL levels were significantly higher in these three groups than in the NIMPN and HC groups. The CSF NFL level was significantly higher in the NIMPN group than in the HC group (Table 2 and Figure 1).

\section{Associations Between NFL Levels and ALS Clinical Parameters}

Using Spearman correlation analysis, there were no significant correlations between NFL level and age at onset, onset site, diagnostic category, clinical phenotype, TTG, or FVC. A significant negative correlation was found

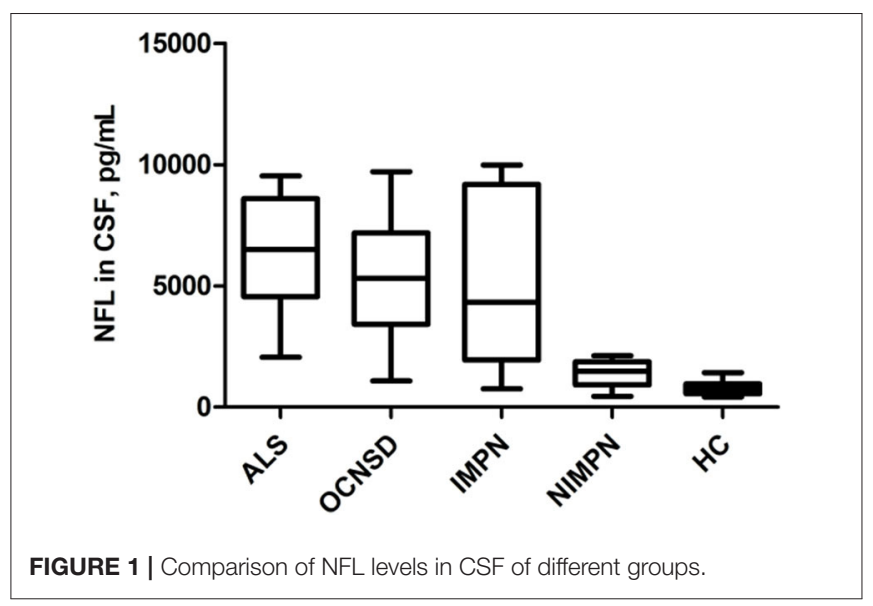

TABLE 3 | Correlations between the levels of NFL and ALS clinical parameters.

\begin{tabular}{lcc}
\hline Clinical parameters & $\boldsymbol{R}$ & $\boldsymbol{p}$-value \\
\hline Age at onset & -0.133 & 0.383 \\
Site of onset & -0.201 & 0.185 \\
Disease duration & -0.190 & 0.212 \\
Diagnostic category & -0.060 & 0.695 \\
Clinical phenotype & -0.050 & 0.743 \\
TTG & -0.339 & 0.123 \\
FVC & -0.154 & 0.350 \\
ALSFRS-r & $-\mathbf{0 . 3 8 8}$ & $\mathbf{0 . 0 0 9}$ \\
DPR & $\mathbf{0 . 4 6 6}$ & $\mathbf{0 . 0 0 1}$ \\
\hline
\end{tabular}

ALS, amyotrophic lateral sclerosis; NFL, neurofilament light chain; Disease duration, time from symptom onset to sample collection; ALSFRS-r, revised ALS functional rating scale; $D P R$, disease progression rate; FVC, forced vital capacity; TTG, the time of symptoms spreading from spinal or bulbar localization to generalization.

The values represent the correlations between NFL level and each clinical parameter. Correlations were considered significant at $p<0.05$. The $p<0.05$ was in bold.

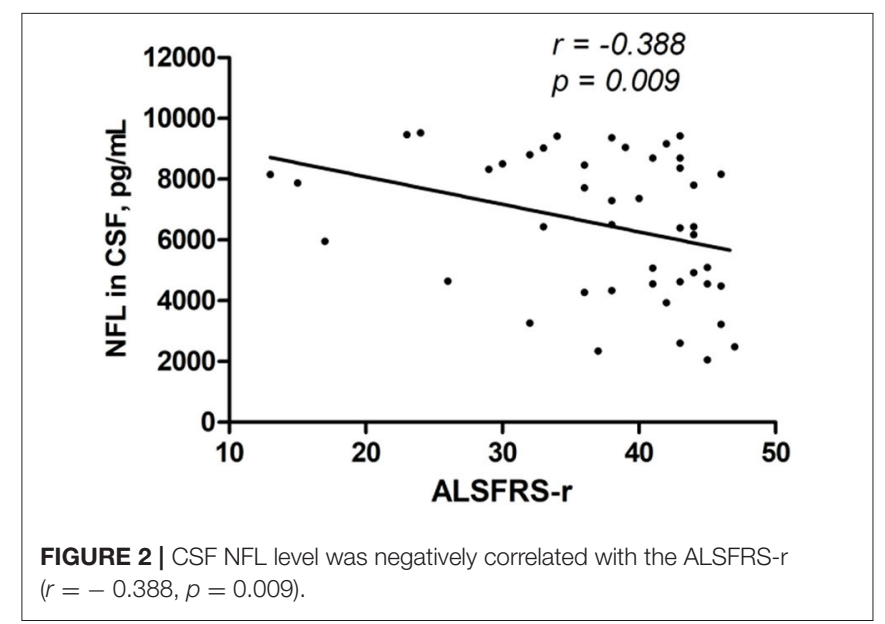

between NFL level and ALSFRS-r and a significant positive correlation between NFL level and DPR (Table 3 and Figures 2, 3). 


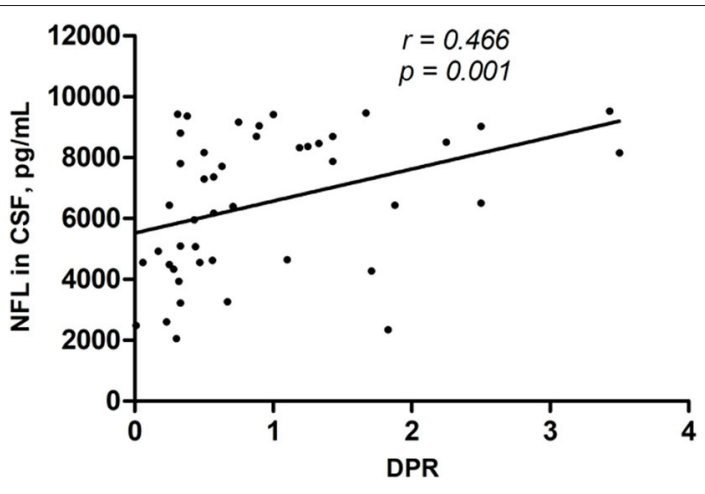

FIGURE 3 | CSF NFL level was positively correlated with DPR $(r=0.466, p=0.001)$.

\section{DISCUSSION}

In this prospective study, the CSF NFL levels were significantly higher in patients with sALS than healthy controls, but were not significantly elevated compared with patients with OCNSD or IMPN, which are expected to lead to significant axon damage. We confirmed that NFL levels are promising prognostic biomarkers for monitoring disease severity and disease progression of sALS.

In recent years, there has been strong evidence from independent groups, large cohorts of patients, and multicenter studies that neurofilament light chains are potentially valuable ALS biomarkers $(3,5-13,21,23)$. Verde et al. showed that serum NFL is increased in ALS compared to other conditions and can serve as a diagnostic and prognostic biomarker using ultrasensitive single-molecule array technology (6). Gaiani et al. confirmed the role of NFL as a diagnostic and prognostic biomarker in ALS, and suggested that elevated CSF NFL levels in patients with upper motor neuron involvement and FTD reflect corticospinal tract degeneration, while low NFL levels in patients with lower motor neuron signs indicate milder disease phenotypes (7). In a multicenter study, Feneberg et al. showed that CSF and serum NF concentrations discriminate ALS with early symptom onset from other neurological diseases (9). In a relatively large cohort of 190 ALS patients and 130 with other neurological diseases, Rossi et al. confirmed that CSF NFL and $\mathrm{p}-\mathrm{NFH}$ were valuable diagnostic and prognostic biomarkers in ALS (10). A prospective, multicenter, longitudinal study validated NfL values as prognostic biomarker for ALS, comparing serum and CSF NfL values (13). Tortelli et al. found that CSF NFL levels were increased in ALS compared to other neurodegenerative disorders and peripheral neuropathies and were correlated with disease severity and progression (23). Contrasting these studies, we found that CSF NFL levels were significantly elevated not only in sALS patients but also in the OCNSD and IMPN groups compared to healthy controls. Comparing ALS, OCNSD, and IMPN patients, the ability of CSF NFL to discriminate groups was low and did not differ significantly. It seems CSF NFLs are non-specific markers present in various neurological diseases that cause significant axon damage. Considering the small number of cases include of our study compared to previous research, a large sample size may be more convincing. Increased CSF NFL levels in patients with CIDP, GBS, or IMPN may be due to the release of axonal protein after nerve root injury (34). Elevated CSF NFL levels in OCNSDs, such as MSA, SCD, and MS, may reflect different degrees of pyramidal tract damage. Furthermore, we observed that the CSF NFL levels in NIMPN were higher than that in HCs and lower than in the IMPN group. We speculated that the NFL concentration in CSF released after nerve root injury is higher than that induced by peripheral nerve injury.

We found a positive correlation between CSF NFL levels and the rate of disease progression and a significant negative correlation between CSF NFL levels and ALS function scores. The rate of disease progression based on the ALS function score is an important indicator of the clinical progression and deterioration of ALS patients (33). Our work is in line with reports that CSF NFL performs relatively well as a prognostic biomarker for ALS. In our ALS sample, we did not find a significant correlation between the NFL concentration and TTG, another disease progression indicator, while another study found a short TTG in patients with high NFL levels (3). This is likely because only 22 of our patients have progressed to spinal and bulbar involvement and have complete TTG data; a small sample size cannot reflect the relationship between the CSF NFL concentration and TTG well. Some studies have also suggested that the CSF NFL levels are higher in ALS patients with predominantly upper motor neuron involvement $(7,22)$, which we did not observe. Of the 45 sALS patients in our study, only four had UMND-ALS. The relatively small proportion may be one of the causes of the phenomenon. In addition, the extent of pyramidal tract damage in classic ALS may not be less than that of prevalent upper motor neuron ALS.

There were some shortcomings to our work. First, the sample size is relatively small; a large sample size may be more convincing. Second, we assayed only the CSF NFL concentration, and not that in blood. A comparison of blood and CSF NFL levels in patients may better explain the results. In addition, serum measurements are more favorable to monitor disease progression, and the reliability of NFL assay also in serum was widely reported in literature. Third, all patients in our study underwent therapies (Riluzole or Edaravone) at the time of lumbar puncture. We did not record the detailed treatment process, which possibly influenced axonal preservation or damage, and future study should consider it.

In conclusion, the CSF NFL levels are increased in patients with sALS and other nervous system diseases. The high CSF NFL levels of sALS patients were associated with more rapidly evolving disease and more severe disability due to ALS.

\section{DATA AVAILABILITY STATEMENT}

The raw data supporting the conclusions of this article will be made available by the authors, without undue reservation. 


\section{ETHICS STATEMENT}

The studies involving human participants were reviewed and approved by the ethics committee of the Chinese PLA General Hospital. The patients/participants provided their written informed consent to participate in this study.

\section{AUTHOR CONTRIBUTIONS}

QS was responsible for the design of the subject, the implementation of the experiment, and the writing of the article. XZ was responsible for the collection of sample. SL was responsible for the guidance of the experiment. FY, HW, and FC were responsible for the guidance of the subject. $\mathrm{XH}$ was in

\section{REFERENCES}

1. Bilsland LG, Sahai E, Kelly G, Golding M, Greensmith L, Schiavo G. Deficits in axonal transport precede ALS symptoms in vivo. Proc Natl Acad Sci USA. (2010) 107:20523-8. doi: 10.1073/pnas.1006869107

2. Gagliardi D, Meneri M, Saccomanno D, Bresolin N, Comi GP, Corti S. Diagnostic and prognostic role of blood and cerebrospinal fluid and blood neurofilaments in amyotrophic lateral sclerosis: a review of the literature. Int J Mol Sci. (2019) 20:4152. doi: 10.3390/ijms20174152

3. Tortelli R, Copetti M, Ruggieri M, Cortese R, Capozzo R, Leo A, et al. Cerebrospinal fluid neurofilament light chain levels: marker of progression to generalized amyotrophic lateral sclerosis. Eur J Neurol. (2015) 22:215-8. doi: 10.1111 /ene. 12421

4. King AE, Blizzard CA, Southam KA, Vickers JC, Dickson TC. Degeneration of axons in spinal white matter in G93A mSOD1 mouse characterized by NFL and $\alpha$-internexin immunoreactivity. Brain Res. (2012) 1465:90-100. doi: 10.1016/j.brainres.2012.05.018

5. Forgrave LM, Ma M, Best JR, DeMarco ML. The diagnostic performance of neurofilament light chain in CSF and blood for Alzheimer's disease, frontotemporal dementia, and amyotrophic lateral sclerosis: a systematic review and meta-analysis. Alzheimers Dement. (2019) 11:730-43. doi: 10.1016/j.dadm.2019.08.009

6. Verde F, Steinacker P, Weishaupt JH, Kassubek J, Oeckl P, Halbgebauer $\mathrm{S}$, et al. Neurofilament light chain in serum for the diagnosis of amyotrophic lateral sclerosis. J Neurol Neurosurg Psychiatr. (2019) 90:157-64. doi: 10.1136/jnnp-2018-318704

7. Gaiani A, Martinelli I, Bello L, Querin G, Puthenparampil M, Ruggero S, et al. Diagnostic and prognostic biomarkers in amyotrophic lateral sclerosis: neurofilament light chain levels in definite subtypes of disease. JAMA Neurol. (2017) 74:525-32. doi: 10.1001/jamaneurol.2016.5398

8. Steinacker P, Huss A, Mayer B, Grehl T, Grosskreutz J, Borck G, et al. Diagnostic and prognostic significance of neurofilament light chain NF$\mathrm{L}$, but not progranulin and $\mathrm{S} 100 \mathrm{~B}$, in the course of amyotrophic lateral sclerosis: Data from the German MND-net. Amyotroph Lateral Scler Frontotemporal Degener. (2017) 18:112-9. doi: 10.1080/21678421.2016.12 41279

9. Feneberg E, Oeckl P, Steinacker P, Verde F, Barro C, Van Damme $\mathrm{P}$, et al. Multicenter evaluation of neurofilaments in early symptom onset amyotrophic lateral sclerosis. Neurology. (2018) 90:e22-30. doi: 10.1212/WNL.0000000000004761

10. Rossi D, Volanti P, Brambilla L, Colletti T, Spataro R, La Bella V. CSF neurofilament proteins as diagnostic and prognostic biomarkers for amyotrophic lateral sclerosis. J Neurol. (2018) 265:510-21. doi: 10.1007/s00415-017-8730-6

11. Steinacker P, Feneberg E, Weishaupt J, Brettschneider J, Tumani H, Andersen PM, et al. Neurofilaments in the diagnosis of motoneuron diseases: a prospective study on 455 patients. J Neurol Neurosurg Psychiatr. (2016) 87:12-20. doi: 10.1136/jnnp-2015-311387 charge of the project, responsible for the project's funding, design, and checking of the article.

\section{FUNDING}

The authors have no actual or potential conflicts of interest. The study was supported by grants from the National Natural Science Foundation of China (Nos. 81671278 and 81601096).

\section{ACKNOWLEDGMENTS}

The authors thank the Chinese PLA General Hospital which has provided materials essential for this study and all the neurologists of the Neurology Department for the support in this study.

12. Li DW, Ren H, Jeromin A, Liu M, Shen D, Tai H, et al. Diagnostic performance of neurofilaments in Chinese patients with amyotrophic lateral sclerosis: a prospective study. Front Neurol. (2018) 9:726. doi: 10.3389/fneur.2018.00726

13. Benatar M, Zhang L, Wang L, Granit V, Statland J, Barohn R, et al. Validation of serum neurofilaments as prognostic and potential pharmacodynamic biomarkers for ALS. Neurology. (2020) 95:e59-69. doi: 10.1212/WNL.0000000000009559

14. Mattsson N, Cullen NC, Andreasson U, Zetterberg H, Blennow K. Association between longitudinal plasma neurofilament light and neurodegeneration in patients with Alzheimer disease. JAMA Neurol. (2019) 76:791-9. doi: 10.1001/jamaneurol.2019.0765

15. Mattsson N, Andreasson U, Zetterberg H, Blennow K, Alzheimer's disease neuroimaging initiative. association of plasma neurofilament light with neurodegeneration in patients with alzheimer disease. JAMA Neurol. (2017) 74:557-66. doi: 10.1001/jamaneurol.2016.6117

16. Norgren N, Sundström P, Svenningsson A, Rosengren L, Stigbrand T, Gunnarsson M. Neurofilament and glial fibrillary acidic protein in multiple sclerosis. Neurology. (2004) 63:1586-90. doi: 10.1212/01.WNL.0000142988.49341.D1

17. Pawlitzki M, Schreiber S, Bittner D, Kreipe J, Leypoldt F, Rupprecht $\mathrm{K}$, et al. CSF neurofilament light chain levels in primary progressive MS: signs of axonal neurodegeneration. Front Neurol. (2018) 9:1037. doi: 10.3389/fneur.2018.01037

18. Abdo WF, Bloem BR, Van Geel WJ, Esselink RA, Verbeek MM. CSF neurofilament light chain and tau differentiate multiple system atrophy from Parkinson's disease. Neurobiol Aging. (2007) 28:742-7. doi: 10.1016/j.neurobiolaging.2006.03.010

19. Sandelius Å, Zetterberg H, Blennow K, Adiutori R, Malaspina A, Laura $M$, et al. Plasma neurofilament light chain concentration in the inherited peripheral neuropathies. Neurology. (2018) 90:e518-24. doi: 10.1212/WNL.0000000000004932

20. De Marchis GM, Katan M, Barro C, Fladt J, Traenka C, Seiffge DJ, et al. Serum neurofilament light chain in patients with acute cerebrovascular events. Eur J Neurol. (2018) 25:562-8. doi: 10.1111/ene.13554

21. Reijn TS, Abdo WF, Schelhaas HJ, Verbeek MM. CSF neurofilament protein analysis in the differential diagnosis of ALS. J Neurol. (2009) 256:615-9. doi: 10.1007/s00415-009-0131-z

22. Menke RA, Gray E, Lu CH, Kuhle J, Talbot K, Malaspina A, et al. CSF neurofilament light chain reflects corticospinal tract degeneration in ALS. Ann Clin Transl Neurol. (2015) 2:748-55. doi: 10.1002/acn3.212

23. Tortelli R, Ruggieri M, Cortese R, D’Errico E, Capozzo R, Leo A, et al. Elevated cerebrospinal fluid neurofilament light levels in patients with amyotrophic lateral sclerosis: a possible marker of disease severity and progression. Eur J Neurol. (2012) 19:1561-7. doi: 10.1111/j.1468-1331.2012.03777.x

24. Gille B, De Schaepdryver M, Goossens J, Dedeene L, De Vocht J, Oldoni E, et al. Serum neurofilament light chain levels as a marker of upper motor neuron degeneration in patients with Amyotrophic Lateral Sclerosis. Neuropathol Appl Neurobiol. (2019) 45:291-304. doi: 10.1111/nan.12511 
25. Lu CH, Macdonald-Wallis C, Gray E, Pearce N, Petzold A, Norgren $\mathrm{N}$, et al. Neurofilament light chain: a prognostic biomarker in amyotrophic lateral sclerosis. Neurology. (2015) 84:2247-57. doi: 10.1212/WNL.0000000000001642

26. De Schaepdryver M, Lunetta C, Tarlarini C, Mosca L, Chio A, Van Damme P, et al. Neurofilament light chain and $\mathrm{C}$ reactive protein explored as predictors of survival in amyotrophic lateral sclerosis. J Neurol Neurosurg Psychiatry. (2020) 91:436-7. doi: 10.1136/jnnp-2019-322309

27. Thouvenot E, Demattei C, Lehmann S, Maceski-Maleska A, Hirtz C, JuntasMorales R, et al. Serum neurofilament light chain at time of diagnosis is an independent prognostic factor of survival in amyotrophic lateral sclerosis. Eur J Neurol. (2020) 27:251-7. doi: 10.1111/ene.14063

28. Brooks BR, Miller RG, Swash M, Munsat TL, World Federation of Neurology Research Group on Motor Neuron Diseases. El Escorial revisited: revised criteria for the diagnosis of amyotrophic lateral sclerosis. Amyotroph Lateral Scler Other Motor Neuron Disord. (2000) 1:293-9. doi: 10.1080/146608200300079536

29. Anderson KJ, Scheff SW, Miller KM, Roberts KN, Gilmer LK, Yang C, et al. The phosphorylated axonal form of the neurofilament subunit NF-H (pNF$\mathrm{H})$ as a blood biomarker of traumatic brain injury. J Neurotrauma. (2008) 25:1079-85. doi: 10.1089/neu.2007.0488

30. Tortelli R, Copetti M, Panza F, Cortese R, Capozzo R, D’Errico E, et al. Time to generalisation as a predictor of prognosis in amyotrophic lateral sclerosis. J Neurol Neurosurg Psychiatry. (2016) 87:678-9. doi: 10.1136/jnnp-2014-308478

31. Strong M, Rosenfeld J. Amyotrophic lateral sclerosis: a review of current concepts. Amyotroph Lateral Scler Other Motor
Neuron Disord. (2003) 4:136-43. doi: 10.1080/146608203100 11250

32. Cedarbaum JM, Stambler N, Malta E, Fuller C, Hilt D, Thurmond $\mathrm{B}$, et al. The ALSFRS-R: a revised ALS functional rating scale that incorporates assessments of respiratory function. BDNF ALS Study Group (Phase III). J Neurol Sci. (1999) 169:13-21. doi: 10.1016/S0022-510X(99)00 210-5

33. Gordon PH, Cheung YK. Progression rate of ALSFRS$\mathrm{R}$ at time of diagnosis predicts survival time in ALS. Neurology. (2006) 67:1314-5. doi: 10.1212/01.wnl.0000243812.2 5517.87

34. Petzold A, Brettschneider J, Jin K, Keir G, Murray NM, Hirsch NP, et al. CSF protein biomarkers for proximal axonal damage improve prognostic accuracy in the acute phase of Guillain-Barré syndrome. Muscle Nerve. (2009) 40:42-9. doi: 10.1002/mus.21239

Conflict of Interest: The authors declare that the research was conducted in the absence of any commercial or financial relationships that could be construed as a potential conflict of interest.

Copyright (c) 2020 Sun, Zhao, Li, Yang, Wang, Cui and Huang. This is an open-access article distributed under the terms of the Creative Commons Attribution License (CC $B Y)$. The use, distribution or reproduction in other forums is permitted, provided the original author(s) and the copyright owner(s) are credited and that the original publication in this journal is cited, in accordance with accepted academic practice. No use, distribution or reproduction is permitted which does not comply with these terms. 